\title{
Boundary states and black hole entropy
}

\author{
J. David Brown \\ Department of Physics and Department of Mathematics, North Carolina State University, Raleigh, North Carolina 27695-8202
}

(Received 22 September 1997; published 16 January 1998)

\begin{abstract}
A formal derivation of black hole entropy is given as a sum over boundary states. The boundary states are labeled by energy and momentum surface densities, and parametrized by the boundary metric. The sum over state labels is expressed as a functional integral with measure determined by the density of states. The sum over metrics is expressed as a functional integral with measure determined by the universal expression for the inverse temperature gradient at the horizon. The analysis applies to any stationary, nonextreme black hole in any theory of gravitational and matter fields.
\end{abstract}

[S0556-2821(98)04704-3]

PACS number(s): 04.70.Dy, 04.20.Fy, 04.60.Gw

Many researchers have suggested that black hole entropy arises from a sum over boundary (or horizon or surface or edge) states [1]. The formal analysis presented in this paper provides strong evidence for the correctness of this conjecture. I consider a system of gravitational and matter fields with outer and inner boundary elements, denoted $B$ and $B^{\prime}$ respectively. The entropy for stationary, nonextreme black holes is derived by summing over all states on the inner boundary element. The states on $B^{\prime}$ are labeled by the energy surface density $\varepsilon^{\prime}$ and momentum surface density $j_{i}^{\prime}$ [2], and parametrized by the surface metric $\sigma_{a b}^{\prime}$. The measure for the integration over $\varepsilon^{\prime}$ and $j_{i}^{\prime}$ is determined by the density of states, which is calculated formally as a path integral. Integration over $\varepsilon^{\prime}$ and $j_{i}^{\prime}$ imposes the condition that $B^{\prime}$ is the bifurcation surface of a stationary black hole horizon. The measure for the integration over $\sigma_{a b}^{\prime}$ is not fixed by the density of states and requires further physical input for its determination. The needed physical input is the inverse temperature gradient at the horizon. The inverse temperature gradient is a universal quantity, in the sense that it is independent of the details of the black hole and independent of the theory of gravity under consideration. In this regard, its input into the calculation is analogous to the identification of inverse temperature with imaginary time period.

Recent results indicate that black hole entropy can be understood in terms of D-brane states in string theory [3]. Although the string theory description of black hole entropy is quite compelling, it is limited in scope to a particular theory of gravity (as defined by the low energy limit of string theory). On the other hand, path integral and variational methods have shown that black hole entropy appears in all diffeomorphism invariant theories of gravitational and matter fields $[4,5]$. This suggests that a deeper understanding of black hole entropy can be found from within the path integral formalism itself, without specialization to a particular theory of gravity. The analysis contained in this paper points to such a deeper understanding by providing an interpretation of the path integral derivation of black hole entropy as a sum over boundary states.

Let me begin by presenting a quick overview of certain key aspects of statistical mechanics. Consider a nongravitating, quantum mechanical system with Hamiltonian operator
$\hat{H}_{V}$ which depends on, say, volume $V$. (For example, the electromagnetic field in a spherical container of volume $V$.) The partition function is defined by $Z_{V}(\beta)=\operatorname{tr} e^{-\beta \hat{H}_{V}}$, where $\beta$ is the inverse temperature. Eigenstates of $\hat{H}_{V}$ are labeled by the corresponding eigenvalue, namely, energy $E$, and are parametrized by volume $V$. The partition function can be written as

$$
Z_{V}(\beta)=\int d E D_{V}(E) e^{-\beta E}
$$

where $D_{V}(E)$ is the density of energy eigenstates. That is, $D_{V}(E) d E$ is the number of states with volume $V$ and energy in the range $d E$. An alternative procedure is to define $Z_{V}(\beta)$ by a path integral over histories that are periodic in imaginary time with period $\beta$. The phase in the path integral is given by the action, which is related to the classical Hamiltonian $H_{V}$ in the usual way. The density of states $D_{V}(E)$ becomes a path integral over histories with fixed energy $E$, where $E$ is the value of the classical Hamiltonian [2].

For a given value of $\beta$, the probability that the system will be found in a state with volume $V$ and energy in the range $d E$ is $\mathcal{P} D_{V}(E) d E$, where $\mathcal{P}=e^{-\beta E} / Z_{V}(\beta)$. The expectation value of energy is defined by $\langle E\rangle=$ $-\partial\left[\ln Z_{V}(\beta)\right] / \partial \beta$, pressure is defined by $\beta p=\partial\left[\ln Z_{V}(\beta)\right] / \partial V$, and entropy is defined by

$$
\mathcal{S}_{V}(\beta)=-\int d E D_{V}(E) \mathcal{P} \ln \mathcal{P}=\ln Z_{V}(\beta)+\beta\langle E\rangle .
$$

This is the entropy of the system in the canonical ensemble at temperature $1 / \beta$. The microcanonical entropy is obtained from the infinite temperature limit, $\beta=0$, in which $\mathcal{P}$ is constant. In this limit Eqs. (1) and (2) yield $\mathcal{S}_{V}(0)$ $=\ln \left[\int d E D_{V}(E)\right]$, which is the logarithm of the number of states with volume $V$. The total number of states $N$, with no restriction on $V$, is given by

$$
N=\int d E d V \mu(V) D_{V}(E) .
$$

Here, $\mu(V)$ is a measure for the $V$ integration that must be specified by some physical criterion. This unspecified mea- 
sure also appears in the definition of the partition function for the constant pressure ensemble [6], namely, $Z_{p}(\beta)$ $=\int d V \mu(V) e^{-\beta p V} Z_{V}(\beta)$. In fact, one can view $N$ as the infinite temperature limit of $Z_{p}(\beta)$.

The density of states factor in Eq. (1) can be written as $e^{\ln \left[D_{V}(E)\right]}$, where for simplicity an arbitrary dimensionful constant (such as the Planck energy) has been omitted from the $\operatorname{logarithm}$. Let us evaluate the integral for $Z_{V}(\beta)$ in the steepest descents approximation. With $E=E^{*}(\beta)$ denoting the solution of the extremum condition $\beta=\partial\left[\ln D_{V}(E)\right] / \partial E$, we find

$$
\ln Z_{V}(\beta) \approx-\beta E^{*}+\ln \left[D_{V}\left(E^{*}\right) \Delta\right]
$$

where $2 \pi \Delta^{-2}=-\left.\left[\partial^{2}\left(\ln D_{V}(E)\right) / \partial(E)^{2}\right]\right|_{E^{*}}$. The steepest descents approximation also yields $\langle E\rangle \approx E^{*}$. By differentiating the relation $\left.\beta \approx\left[\partial\left(\ln Z_{V}(E)\right) / \partial E\right]\right|_{\langle E\rangle}$ with respect to $\beta$, we find that $\Delta^{2}$ is given by the mean square deviation in energy, $\Delta^{2} /(2 \pi) \approx\left\langle(E-\langle E\rangle)^{2}\right\rangle$. A comparison of Eqs. (2) and (4) shows that

$$
\mathcal{S}_{V}(\beta) \approx \ln \left[D_{V}\left(E^{*}\right) \Delta\right]
$$

Thus, the entropy in the canonical ensemble at temperature $1 / \beta$ is the logarithm of the number of states in an energy interval given by the mean square deviation in energy. Equation (5) can be written as $\mathcal{S}_{V}(\beta) \approx \ln \left[D_{V}(E) \Delta\right]$ where $\beta$ and $E$ are related by $\beta=\partial\left[\ln D_{V}(E)\right] / \partial E$.

Now consider vacuum Einstein gravity on a spatial manifold $\Sigma$. The boundary $\partial \Sigma$ need not be simply connectedlater we will specialize to the case in which $\partial \Sigma$ consists of an inner boundary element $B^{\prime}$ and an outer boundary element $B$. The partition function is defined as a path integral on the manifold $\Sigma \times S^{1}$ with action ${ }^{1}$

$$
\begin{gathered}
S^{p f}=i \int d t\left(\int_{\Sigma} d^{3} x P^{i j} \dot{h}_{i j}-H_{\sigma}\right), \\
H_{\sigma}=\int_{\Sigma} d^{3} x\left(N \mathcal{H}+V^{i} \mathcal{H}_{i}\right)+\int_{\partial \Sigma} d^{2} x \sqrt{\sigma}\left(N \varepsilon-V^{i} j_{i}\right),
\end{gathered}
$$

where $h_{i j}$ and $P^{i j}$ are the canonical variables, $N$ and $V^{i}$ are the lapse function and shift vector, and $\mathcal{H}$ and $\mathcal{H}_{i}$ are the Hamiltonian and momentum constraints. Also, $\sigma_{a b}$ is the metric on $\partial \Sigma$ and $\varepsilon=k /(8 \pi)$ and $j_{i}=-2 P_{i j} n^{j} / \sqrt{h}$ are the energy surface density and momentum surface density [2]. Here, $k=\sigma^{a b} k_{a b}$ and $n^{j}$ denote, respectively, the trace of the extrinsic curvature and the outward pointing unit normal of $\partial \Sigma$ embedded in $\Sigma$.

The variation of the action (6) is [2]

\footnotetext{
${ }^{1}$ See Ref. [2]. The action in Eq. (6) is defined by the exponential in the path integral, and therefore equals $i$ times the "Lorentzian action." Also, for simplicity, the condition $V^{i} n_{i}=0$ on $\partial \Sigma$ has been imposed; this means that the boundary is at rest with respect to the constant time slices. The action in the presence of moving boundaries is given in Ref. [7].
}

$$
\begin{aligned}
\delta S^{p f}= & \cdots-i \int d t \int_{\partial \Sigma} d^{2} x \sqrt{\sigma}\left\{\varepsilon \delta N-j_{i} \delta V^{i}\right. \\
& \left.-\left(N s^{a b} / 2\right) \delta \sigma_{a b}\right\},
\end{aligned}
$$

where the unwritten terms yield the classical equations of motion and $\left(N s^{a b} / 2\right)=\left[N k^{a b}+\left(n^{i} \partial_{i} N-N k\right) \sigma^{a b}\right] /(16 \pi)$ defines the spatial stress. The form of $\delta S^{p f}$ shows that $\sigma_{a b}$ and the boundary values of $N$ and $V^{i}$ are held fixed in the variational principle. The partition function, defined by the path integral, is a functional of these quantities. The boundary value of $N$ determines the period in imaginary time, which is identified with the inverse temperature on the system boundary: $\beta=\left.i \int d t N\right|_{\partial \Sigma}$. (Note that $\beta$ is not typically constant on $\partial \Sigma$ due to gravitational redshifting and blueshifting.) The boundary value of $V^{i}$ defines the proper velocity $v^{i}$ of the spatial coordinate system through the relation $\beta v^{i}$ $=\left.i \int d t V^{i}\right|_{\partial \Sigma}$. (In the classical approximation, $v^{i}$ is the spatial velocity of the system with respect to observers at rest on the boundary $[8,2]$.) The partition function will be denoted $Z_{\sigma}[\beta, v]$. To be precise, $Z_{\sigma}[\beta, v]$ is the grand canonical partition function and $v^{i}$ plays the role of the "chemical potential" for $j_{i}$.

Because the Hamiltonian and momentum constraints vanish on shell, the value of the Hamiltonian $H_{\sigma}$ for various choices of lapse and shift is given by the densitized energy surface density $\sqrt{\sigma} \varepsilon$ and the densitized momentum surface density $\sqrt{\sigma} j_{i}$. These quantities play the role of energy. The fact that there are many such "energies" reflects the manyfingered character of time in gravitational physics. The fact that they are surface quantities reflects the fact that the gravitational field on $\partial \Sigma$ is determined by the mass-energy throughout $\Sigma$. By analogy with the nongravitating system discussed above, we are led to the formal identification of $\sqrt{\sigma} \varepsilon$ and $\sqrt{\sigma} j_{i}$ as labels for (boundary) states. These states are parametrized by the boundary metric $\sigma_{a b}$. In direct analogy with Eq. (1), the partition function can be written as

$$
Z_{\sigma}[\beta, v]=\int d \varepsilon d j D_{\sigma}[\varepsilon, j] e^{-\int_{\partial \Sigma} d^{2} x \sqrt{\sigma}\left(\beta \varepsilon-\beta v^{i} j_{i}\right)}
$$

where $\int d \varepsilon d j$ denotes functional integration over $\sqrt{\sigma} \varepsilon$ and $\sqrt{\sigma} j_{i}$. In Eq. (8), $D_{\sigma}[\varepsilon, j]$ is the density of boundary states; that is, $D_{\sigma}[\varepsilon, j] d \varepsilon d j$ is the number of boundary states with metric $\sigma_{a b}$ and energy and momentum surface densities in the range $d \varepsilon d j$.

The entropy of the gravitational field in the canonical ensemble with boundary temperature $1 / \beta$ can be computed from the partition function $Z_{\sigma}[\beta, v]$ using the analog of Eq. (2). Alternatively, one can use the density of states $D_{\sigma}[\varepsilon, j]$ and the analog of Eq. (5). In the zero-loop approximation, in which only the dominant exponential contributions to the path integrals for $Z_{\sigma}[\beta, v]$ and $D_{\sigma}[\varepsilon, j]$ are kept, the second option is easier. Indeed, in this approximation the mean square deviation factor $\Delta$ can be dropped and the analog of Eq. (5) becomes $\mathcal{S}_{\sigma}[\beta, v] \approx \ln \left(D_{\sigma}[\varepsilon, j]\right)$. Here, it is understood that $\beta=\delta\left(\ln D_{\sigma}[\varepsilon, j]\right) / \delta(\sqrt{\sigma} \varepsilon)$ with a similar relation for $\beta v^{i}$. Now, from the path integral for $Z_{\sigma}[\varepsilon, j]$, we find that the density of states is expressed as a path integral over the manifold $\Sigma \times S^{1}$ with action 


$$
S^{d s}=i \int d t \int_{\Sigma} d^{3} x\left(P^{i j} \dot{h}_{i j}-N \mathcal{H}-V^{i} \mathcal{H}_{i}\right)
$$

In the zero-loop approximation, $D_{\sigma}[\varepsilon, j]$ equals the exponential of the action $S^{d s}$ evaluated at the stationary classical solution (if one exists) with the given values of $\varepsilon, j_{i}$, and $\sigma_{a b}$ on $\partial \Sigma$. Since $S^{d s}=0$ for a stationary classical solution, the zero-loop contribution to $D_{\sigma}[\varepsilon, j]$ is 1 and the entropy $\mathcal{S}_{\sigma}[\beta, v] \approx \ln \left(D_{\sigma}[\varepsilon, j]\right)$ vanishes.

The conclusion $\mathcal{S}_{\sigma}[\beta, v] \approx 0$ is general. Note, however, that $\varepsilon$ and $j_{i}$ label the states for the complete boundary $\partial \Sigma$ of the spatial manifold. If $\partial \Sigma$ consists of disconnected pieces, then $\varepsilon$ and $j_{i}$ include labels for states on each boundary element. Likewise, $\beta, v^{i}$, and $\sigma_{a b}$ specify the inverse temperature, velocity, and metric on each boundary element. Let us assume that $\partial \Sigma$ consists of an "outer" boundary element $B$ and an 'inner" boundary element $B^{\prime}$, and make this dependence explicit by writing $D\left[\varepsilon, j ; \sigma \mid \varepsilon^{\prime}, j^{\prime} ; \sigma^{\prime}\right]$ for the density of states and $Z\left[\beta, v ; \sigma \mid \beta^{\prime}, v^{\prime} ; \sigma^{\prime}\right]$ for the partition function. Here, the unprimed quantities refer to $B$ and the primed quantities refer to $B^{\prime}$. The vanishing entropy is expressed as $\mathcal{S}\left[\beta, v ; \sigma \mid \beta^{\prime}, v^{\prime} ; \sigma^{\prime}\right] \approx 0$.

As an example, consider a Schwarzschild black hole. Let the data $\varepsilon, j_{i}, \sigma_{a b}$ on $B$ and the data $\varepsilon^{\prime}, j_{i}^{\prime}, \sigma_{a b}^{\prime}$ on $B^{\prime}$ coincide with those for two " $r=$ const" surfaces. By construction, the classical approximation of the system will consist of the portion of the black hole between the two " $r$ $=$ const" surfaces. According to the analysis above, the entropy of this system vanishes. This is a rather intriguing result when one considers the fact that the boundary elements $B$ and $B^{\prime}$ need not lie in the same wedge of the Kruskal diagram [9]. If $B$ lies in, say, the right wedge and $B^{\prime}$ lies in the left wedge, the system (in the classical approximation) will contain a black hole, yet the entropy will vanish. (Note that, in order for the action (9) and the path integral to be well defined, it is not necessary for the spatial slices of the classical approximation to be nonintersecting.) This, of course, is not the usual result for black hole entropy. In the usual path integral derivation of black hole entropy only the data on a single outer boundary element are specified [2]. Loosely speaking, the information present in the inner boundary data has eliminated the black hole entropy.

Consider, then, our system with inner and outer boundary elements, but with data specified only on the outer boundary element. Let us assume that the outer boundary data are chosen such that the system is classically approximated by a stationary, nonextreme black hole. The entropy of the system is the logarithm of the number of outer boundary states with metric $\sigma_{a b}$ and with $\varepsilon$ and $j_{i}$ in an interval $\Delta$. The number of such states is given by the "density of outer boundary states,"

$$
D[\varepsilon, j ; \sigma]=\int d \varepsilon^{\prime} d j^{\prime} d \sigma^{\prime} \mu\left[\sigma^{\prime}\right] D\left[\varepsilon, j ; \sigma \mid \varepsilon^{\prime}, j^{\prime} ; \sigma^{\prime}\right],
$$

obtained by summing over all inner boundary states. Equation (8) shows that the integration over state labels $\varepsilon^{\prime}$ and $j_{i}^{\prime}$ can be viewed as a transformation to the canonical ensemble with $\beta^{\prime}=0$ and $\beta^{\prime} v^{\prime i}=0$. (One can show that $v^{\prime i}=0$ as well [5].) Thus, the boundary element $B^{\prime}$ becomes an infinite temperature surface. For the black hole that approximates the system, $B^{\prime}$ is the bifurcation surface of the Killing horizon.

In Eq. (10), which is the analog of Eq. (3) with respect to the inner boundary data, a measure factor $\mu\left[\sigma^{\prime}\right]$ appears. This measure can be specified as follows. Consider the path integral for $D\left[\varepsilon, j ; \sigma \mid \varepsilon^{\prime}, j^{\prime} ; \sigma^{\prime}\right]$, constructed from the action $S^{d s}$. In the steepest descents approximation, the integrals in Eq. (10) yield the condition that the variation $\delta\left(S^{d s}\right.$ $\left.+\ln \mu\left[\sigma^{\prime}\right]\right)$ with respect to $\varepsilon^{\prime}, j_{i}^{\prime}$, and $\sigma_{a b}^{\prime}$ should vanish. Equations (6), (7), and (9) show that $\delta\left(S^{d s}+\ln \mu\left[\sigma^{\prime}\right]\right)$ includes the inner boundary terms

$$
\begin{aligned}
& i \int d t \int_{B^{\prime}} d^{2} x\left\{N \delta(\sqrt{\sigma} \varepsilon)-V^{i} \delta\left(\sqrt{\sigma} j_{i}\right)+\sqrt{\sigma}\left(N s^{a b} / 2\right) \delta \sigma_{a b}\right\} \\
& +\int_{B^{\prime}} d^{2} x\left(\delta \ln \mu / \delta \sigma_{a b}\right) \delta \sigma_{a b} .
\end{aligned}
$$

From the first two terms we recover the results $\beta^{\prime}=0$ and $\beta^{\prime} v^{i^{\prime}}=0$. The remaining terms yield (since $\delta \sigma_{a b}$ is $t$-independent) $\delta \ln \mu / \delta \sigma_{a b}=-i \int d t \sqrt{\sigma}\left(N s^{a b} / 2\right)$. With the definition of spatial stress given just after Eq. (7) and the result $\beta^{\prime}=0$, this reduces to $\delta \ln \mu / \delta \sigma_{a b}=$ $-(i / 16 \pi) \int d t \sqrt{\sigma}\left(n^{i} \partial_{i} N\right) \sigma^{a b}$. For the classical extremum, the inverse temperature measured by a stationary observer at any spatial location is given by $\beta=i \int d t N$. Also, $n^{i}$ is $t$-independent. Thus, $i \int d t n^{i} \partial_{i} N=n^{i} \partial_{i} \beta$ and the condition on $\mu$ becomes

$$
\frac{\delta \ln \mu[\sigma]}{\delta \sigma_{a b}}=\frac{1}{16 \pi} \sqrt{\sigma}\left(-n^{i} \partial_{i} \beta\right) \sigma^{a b} .
$$

Now, the factor $-n^{i} \partial_{i} \beta$, which is the inverse temperature gradient at $B^{\prime}$, obeys $-n^{i} \partial_{i} \beta=2 \pi$. This is a universal result, in the sense that it applies to all bifurcate Killing horizons in all theories of gravity. It can be derived, for example, by considering the response of a particle detector that maintains a constant proper distance from the horizon. ${ }^{2}$ Derived in this way, it is apparent that the result does not depend on the particular theory of gravity under consideration. Also it does not depend on the details of the spacetime geometry since, at sufficiently close distances, any bifurcate Killing horizon is physically indistinguishable from a "Rindler horizon" (the horizon in flat spacetime generated by a Lorentz boost). To be specific, then, the desired result can be derived from the expression $\beta=2 \pi / a$ for the inverse temperature measured by a detector undergoing constant acceleration $a$ in

\footnotetext{
${ }^{2}$ The boundary data are stationary and nonsingular, so I will assume that the appropriate quantum state is the stationary Hadamard vacuum [10]. Note that such a state exists only if the Killing vector field is everywhere timelike. For stationary, asymptotically flat black holes such as the Kerr solution, one can possibly define the Hadamard vacuum by taking the outer boundary $B$ to lie inside the speed-of-light surface. Also note that $-n^{i} \partial_{i} \beta$ equals $i \kappa P$, where $\kappa$ is the surface gravity and $P$ is the coordinate time period [5]. If the black hole is asymptotically flat, and if the lapse function is normalized to unity at infinity, then $i P$ equals the inverse temperature $\beta_{\infty}$ at infinity. Under these conditions the result $-n^{i} \partial_{i} \beta=2 \pi$ is equivalent to $\beta_{\infty}=2 \pi / \kappa$.
} 
the Minkowski vacuum of flat spacetime [10]. It is not difficult to show that $1 / a$ measures proper radial distance from the bifurcation surface. Hence, we have $-n^{i} \partial_{i} \beta=2 \pi$ and the condition on the measure $\mu[\sigma]$ becomes $\delta \ln \mu[\sigma] / \delta \sigma_{a b}$ $=\sqrt{\sigma} \sigma^{a b} / 8$. Integrating this expression, we find that $\ln \mu\left[\sigma^{\prime}\right]=\int_{B^{\prime}} d^{2} x \sqrt{\sigma} / 4$.

With the measure $\mu\left[\sigma^{\prime}\right]$ and the previous result $D\left[\varepsilon, j ; \sigma \mid \varepsilon^{\prime}, j^{\prime} ; \sigma^{\prime}\right] \approx 1$, the density of outer boundary states (10) becomes $D[\varepsilon, j ; \sigma] \approx \exp (A / 4)$. Here, $A=\int_{B^{\prime}} d^{2} x \sqrt{\sigma}$ is the horizon area of the black hole that approximates the system. The entropy is then $\mathcal{S}[\beta, v ; \sigma] \approx \ln D[\varepsilon, j ; \sigma] \approx A / 4$ as expected.

The above reasoning can be used to derive the black hole entropy in any diffeomorphism invariant theory of gravitational and matter fields. Start with the general action $S$ of Refs. [4,5]. $S$ is the integral over spacetime $\mathcal{M}$ of a Lagrangian density $\mathcal{L}$. Assume that a boundary term can be added to $S$ to yield an action $S^{p f}$ whose fixed boundary data include the induced metric on $\partial \mathcal{M}$. The path integral with action $S^{p f}$ is the partition function. As in Eq. (7), the variation of $S^{p f}$ defines the state labels $\varepsilon, j_{i}$, as well as the spatial stress $s^{a b}$. As emphasized in Ref. [5], the action $S^{d s}$ for the density of states contains no boundary terms when written in Hamiltonian form. Thus, $\delta S^{d s}$ contains no variations of $N$ or $V^{i}$ on $\partial \Sigma$, and $S^{d s}$ must differ from $S^{p f}$ by boundary terms that change the fixed boundary data from $N, V^{i}$, and $\sigma_{a b}$ to $\varepsilon, j_{i}$, and $\sigma_{a b}$. In particular, $\delta S^{d s}$ must include boundary terms of the same form as those that appear in the first integral of Eq. (11).

Black hole entropy is derived by summing over inner boundary states to form the density of outer boundary states, as in Eq. (10). Integration over $\varepsilon^{\prime}$ and $j_{i}^{\prime}$ implies that the inner boundary element $B^{\prime}$ is the bifurcation surface of a black hole horizon. Integration over other state labels implies that the products of $\beta$ with certain "intensive" variables should vanish at $B^{\prime}[5]$. The measure $\mu\left[\sigma^{\prime}, \ldots\right]$ depends on the surface metric $\sigma_{a b}^{\prime}$ and other parameters. It is obtained, as in Eq. (11), from the requirement that $\delta\left(S^{d s}\right.$ $+\ln \mu$ ) should vanish for variations in $\sigma_{a b}^{\prime}$. The spatial stress $\left(N s^{a b} / 2\right)$ is needed for this calculation, and is obtained from the following considerations. The actions $S$ and $S^{d s}$ differ by boundary terms. In Ref. [5] it is shown that one of these boundary terms has integrand $-4 \sqrt{\sigma} n_{i} U_{0}^{\perp i j \perp} \partial_{j} N$, and the other boundary terms are linear in the (undifferentiated) lapse $N$ or shift $V^{i}$. Here, $U_{0}^{\mu \nu \rho \sigma}$ is the variational derivative of $\mathcal{L}$ with respect to the Riemann tensor $\mathcal{R}_{\mu \nu \rho \sigma}$. Also, $\perp$ designates a component in the $u_{\mu}$ direction, where $u_{\mu}$ is orthogonal to $\Sigma$. Now, $\delta S$ contains only boundary terms at $\partial \mathcal{M}$. If the lapse on an element of $\partial \Sigma$, say $B^{\prime}$, vanishes, then $B^{\prime}$ is a surface where the foliation degenerates. In this case the only possible contribution at $B^{\prime}$ to the boundary terms of $\delta S$ is a "corner" term proportional to $\delta\left(\partial_{i} N\right)$ [7]. On the other hand, we see that $\delta\left(S-S^{d s}\right)$ includes a term $\int_{B^{\prime}}$ $\left(-2 \sqrt{\sigma} n_{i} U_{0}^{\perp i j \perp} \partial_{j} N\right) \sigma^{a b} \delta \sigma_{a b}$. This is not a corner term, so it must come from $\delta S^{d s}$. Therefore the spatial stress, which appears in the boundary terms of $\delta S^{d s}$, must be given by $\left(N s^{a b} / 2\right)=\left(2 n_{i} U_{0}^{\perp i j \perp} \partial_{j} N\right) \sigma^{a b}+\cdots$. Here, the unwritten terms contain undifferentiated linear factors of $N$ or $V$. The condition on the measure that follows from Eq. (11) is then $2 \sqrt{\sigma} n_{i} U_{0}^{\perp i j \perp} n_{j}\left(-n^{k} \partial_{k} \beta\right) \sigma^{a b}=\delta \ln \mu[\sigma, \ldots] / \delta \sigma_{a b}$, where $\partial_{i} N$ is proportional to $n_{i}$ [5]. With the universal result $-n^{k} \partial_{k} \beta=2 \pi$ for the inverse temperature gradient, we find that to leading order the measure is given by $\ln \mu\left[\sigma^{\prime}, \ldots\right]$ $=8 \pi \int_{B^{\prime}} \sqrt{\sigma} n_{i} U_{0}^{\perp i j \perp} n_{j}$. This yields the desired result [4,5], $\mathcal{S}[\beta, v ; \sigma] \approx \ln D[\varepsilon, j ; \sigma] \approx-2 \pi \int_{B^{\prime}} \sqrt{\sigma} \epsilon_{\mu \nu} U_{0}^{\mu \nu \rho \sigma} \epsilon_{\rho \sigma}$, where $\epsilon_{\mu \nu}=2 u_{[\mu} n_{\nu]}$ is the binormal of the bifurcation surface $B^{\prime}$.

I would like to thank J. W. York for valuable insights.
[1] See A. P. Balachandran, L. Chandar, and A. Momen, Nucl. Phys. B461, 581 (1996); J. C. Baez, J. P. Muniain, and D. D. Píriz, Phys. Rev. D 52, 6840 (1995); L. Smolin, J. Math. Phys. 36, 6417 (1995); C. Teitelboim, Phys. Rev. D 53, 2870 (1996); S. Carlip, ibid. 55, 878 (1997); M. Bañados and A. Gomberoff, gr-qc/9611044; and references therein. Results that are in conflict with this idea have been obtained by J. Gegenberg, G. Kunstatter, and T. Strobl, Phys. Rev. D 55, 7651 (1997).

[2] J. D. Brown and J. W. York, Phys. Rev. D 47, 1407 (1993); 47, 1420 (1993).

[3] See A. Strominger and C. Vafa, Phys. Lett. B 379, 99 (1996);
G. Horowitz, gr-qc/9604051; and references therein.

[4] V. Iyer and R. M. Wald, Phys. Rev. D 50, 846 (1994).

[5] J. D. Brown, Phys. Rev. D 52, 7011 (1995).

[6] T. L. Hill, Statistical Mechanics (McGraw-Hill, New York, 1956); R. A. Sach, Mol. Phys. 2,8 (1959).

[7] J. D. Brown, S. R. Lau, and J. W. York (in preparation).

[8] J. D. Brown, E. A. Martinez, and J. W. York, Phys. Rev. Lett. 66, 2281 (1991).

[9] E. A. Martinez, Phys. Rev. D 51, 5732 (1995).

[10] R. M. Wald, Quantum Field Theory in Curved Spacetime and Black Hole Thermodynamics (University of Chicago Press, Chicago, 1994). 